\title{
SELECTIVE ATTENTION AND COGNITIVE DECLINE IN INSTITUTIONALIZED ELDERLY
}

\author{
R. Almeida ${ }^{1}$, M. Marques ${ }^{1}$, H. Espírito Santo ${ }^{2}$, S. Moitinho ${ }^{1}$, V. Vigário ${ }^{1}$, I. Pena ${ }^{1}$, J. Matreno ${ }^{1}$, F. Rodrigues ${ }^{1}$, E. Antunes ${ }^{1}$, \\ D. Simões ${ }^{1}$, A. Costa $^{1}$, A.R. Correia ${ }^{1}$, A.S. Pimentel ${ }^{1}$, V. Alves ${ }^{1}$, T. Nascimento ${ }^{1}$, M. Costa ${ }^{1}$, M. Tomaz ${ }^{1}$, L. Caldas ${ }^{1}$, L.

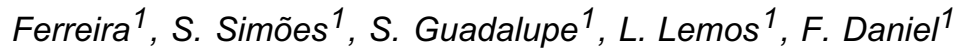 \\ ${ }^{1}$ Instituto Superior Miguel Torga, ${ }^{2}$ CEPESE/Centro de Investigação e Desenvolvimento, Miguel Torga Higher Institute, \\ Coimbra, Portugal
}

Introduction: When cognitive decline (CD) is present, attention is one of the impaired mental functions. CD is also associated with anxious/depressive symptoms and with some demographic variables, particularly, age.

Objectives: Investigate the associations between selective attention (Stroop Test: Stroop_Word, Stroop_Color, Difference between Stroop_Word and Stroop_Color, Stroop Ratio_Word, Stroop Ratio_Color and Difference between Stroop Ratio_Word and Stroop Ratio_ Color) and CD (Montreal Cognitive Assessment/MoCA) in institutionalized elders; explore the predictive value of Stroop variables for $\mathrm{CD}$, controlling anxious/depressive symptoms and sociodemographic variables.

Methods: 140 institutionalized elders (mean age, $\mathrm{M}=78.4, \mathrm{SD}=7.48$, range $=60-97$ ) voluntarily answered to sociodemographic questions, the MoCA, the Geriatric Anxiety Inventory/GAI, the Geriatric Depression Scale/GDS and Stroop test.

Results: 73 elders $(52,1 \%)$ had CD. Dichotomized MoCA was associated with Stroop_Word, Stroop_Color, Stroop Ratio_Word, Stroop Ratio_Color, GDS and the sociodemographic variable schooling $\times$ profession. Age and education were not tested, since MoCA was stratified according to those variables. GDS, Stroop Ratio_Word and Stroop Ratio_Color showed to predict $\mathrm{CD}$.

Conclusions: There was an association between Stroop_Word, Stroop_Color, Stroop Ratio_Word and Stroop Ratio_Color and $C D$, confirming that selective attention is smaller when the elderly reveal CD. GDS and CD were, also, associated. However, there was no association between MoCA dichotomized and differences between the correct answers (Stroop_Word and Stroop_Color) and Ratios (Stroop Ratio_Word and Stroop Ratio_Color). Selective attention and depressive symptoms predicted $\mathrm{CD}$. It would be important to intervene through cognitive rehabilitation with the elders to improve their attention. 